\title{
Medizinstudium und ärztliche Ethik
}

Von H.P. Hartmann

«Die Medizin beschäftigt den ganzen Menschen, weil sie sich mit dem ganzen Menschen beschäftigt.» (Goethe)

\section{Einleitung}

Während seiner theoretischen Ausbildung wird der Medizinstudent vor allem konfrontiert mit den neuesten wissenschaftlichen Methoden und Erkenntnissen. Die notwendigen praktischen Erfahrungen eignet er sich im Wahlstudienjahr als Unterassistent und später im Spital als Assistenzarzt an. Neben dem Wissen und der praktischen Erfahrung macht den wahren Arzt aber in erster Linie sein humanitäres Verhalten aus. Arztsein aus Beruf und Arztsein aus Berufung sind zwei verschiedene Dinge ${ }^{21}$. Karl Jaspers spricht von den zwei Säulen des ärztlichen Handelns, einerseits der naturwissenschaftlichen Erkenntnis und dem technischen Können mit der Basis in der Antike, andererseits dem Ethos der Humanität, wie es von den Mönchen des Mittelalters gepflegt wurde ${ }^{9}$. Diese Humanität, das mitmenschliche Verhältnis zu seinem Gegenüber, die Achtung vor dem Kranken als Person und Persönlichkeit geht vor die wissenschaftliche Erkenntnis und die intuitive Schau des Arztes. Sie ist das oberste Gebot des Arztes; alles andere steht zurück ${ }^{13}$. Der Satz «salus aegroti suprema lex» ist die ärztliche Formulierung des allgemeinen Gebotes der Nächstenliebe und offenbart die entscheidende Formung des abendländischen Arztes durch das Christentum. Adolf Laufs weist zurecht darauf hin, daß das Fremdwort «therapieren» im ursprünglichen Wortsinn «dienen» bedeutet, das Wort «kurieren» der waltenden Fürsorge entspricht ${ }^{11}$. Humanität und christliche Nächstenliebe umspannen einen wesentlichen Teil der ärztlichen Ethik; ihre Wurzeln findet sie im Eid des Hippokrates, der in den letzten Jahren wieder eine eigentliche Renaissance erlebt. Es ist bezeichnend, daß in unserer modernen, materialistischen Zeit eine intensive Rückbesinnung auf die ärztliche Ethik erfolgt, was sich durch zahlreiche Artikel sowohl in der Fachpresse als auch in Laien-Veröffentlichungen manifestiert ${ }^{7,22,26}$. Ganze Bücher widmen sich ausschließlich Fragen der ärztlichen Ethik ${ }^{6,20}$. Möglicherweise spiegelt sich in diesem Bemühen die Angst vor dem Verlust der Grundlagen unseres Berufsstandes im Zusammenhang mit den Fortschritten der Medizintechnik 
und den Veränderungen der neuen Gesellschaftsmoral (Beispiele: Möglichkeit von tiefgreifenden Eingriffen in die körperliche, psychische und seelische Sphäre einer Person; Verwendung von Menschen oder Tieren als Forschungsobjekte; Abwertung der Ehrfurcht vor dem keimenden und dem erlöschenden Leben). In verschiedenen Ländern, so unter anderem auch in der Schweiz, haben sich die medizinischen Dachvereinigungen verpflichtet gefühlt, Grundsätze zur ärztlichen Ethik zu schaffen, um dem Arzt eine Leitlinie für sein Handeln zu geben ${ }^{14}$.

\section{Ethik während der Ausbildung}

Es gibt verschiedene Meinungen zur Frage, zu welchem Zeitpunkt ein Medizinstudent mit Problemen der ärztlichen Ethik sinnvoll konfrontiert werden kann. Es wäre meines Erachtens unrichtig, ihm bei Studienbeginn philosophische Vorlesungen anzubieten. Diese Aufgabe soll vielmehr den Mittelschulen überlassen bleiben und gehört dort zur Vermittlung einer guten Allgemeinbildung. Das Medizinstudium, einerseits gestrafft und gekürzt durch die neueste Studienreform, andererseits gezwungen, ständig die neu entdeckten Erfahrungen und Erkenntnisse darzubieten, muß sich als Fachausbildung auf die wesentlichen Grundlagen der Schulmedizin und ihrer Fortschritte beschränken. Eine zusätzliche Pflichtvorlesung für medizinische Ethik käme wahrscheinlich einer vom Studenten kaum verstandenen Belastung gleich. Vielmehr soll deshalb versucht werden, laufend ärztlich-ethische Fragen in die einzelnen Vorlesungen und praktischen Kurse einzubauen. Dazu bieten sich ständig Gelegenheiten. Voraussetzung ist ein überzeugtes und sicheres ethisches Empfinden des Lehrers und sein Bestreben, neben den Fachkenntnissen Menschlichkeit und Ehrfurcht vor den Geheimnissen der Natur und des Lebens zu vermitteln. Betrachten wir den Ablauf des Medizinstudiums in den einzelnen Phasen, so lassen sich eine ganze Reihe von Beispielen ärztlicher Ethik in den verschiedenen Ausbildungsstufen überlegen:

\section{Naturwissenschaftliche Grundlagen (1. Studienjahr)}

Die Exaktheit und Folgerichtigkeit des chemischen und physikalischen Experiments sowie die Vielfalt und Mannigfaltigkeit im Bereich der Biologie soll dem Studierenden neben der Wissensvermittlung und Anleitung zu 
genauem wissenschaftlichem Versuch auch das Staunen über die Wunder der Natur offenbaren, so daß er angeregt wird zu Gedanken über Ursprung und Entwicklung unserer Welt (Schöpfung und sinnvolle überirdische Lenkung).

\section{Bau und Funktion des Menschen (1. und 2.Studienjahr)}

Die Einblicke in die makroskopische und mikroskopische Morphologie sowie in die komplexen Zusammenhänge der physiologischen und biochemischen Abläufe gestatten es den Dozenten, sowohl bei den Vorlesungen als auch in den Experimenten Entdeckerfreude und Ehrfurcht vor dem menschlichen Körper und seinen Organen zu wecken. Der Tierversuch als Modellversuch soll neben seinem Sinn und seiner Notwendigkeit gleichzeitig auch die Rücksichtnahme auf jede lebende Kreatur aufzeigen. Im Studienplan werden dem Studenten schließlich Einführungen in das Gebiet der Psychologie und der Psychiatrie angeboten, bei denen automatisch eine Reihe ethischer Fragen auftaucht.

\section{Grundfächer und Klinik (in Zürich 3., 4. und 6. Studienjahr)}

Das Studium der Krankheit steht sowohl in den Magistralvorlesungen als auch in den praktischen Kursen im Vordergrund. Dennoch darf dabei der kranke Mensch nicht vergessen werden. Der Lehrer halte sich vor Augen, daß ein Krankheitsbild mit seinen charakteristischen Symptomen einem Studenten weit besser in Erinnerung bleibt, wenn es ihm in der Person eines Leidenden vorgezeigt wird und er sich gleichzeitig der Gefühle und Reaktionen des Kranken auf sein Leiden gewahr wird (Schmerz, Trauer, Verzweiflung, Hoffnung, Vertrauen, Zuversicht). Dies ist auch der Grund, weshalb beispielsweise audio-visuelle Unterrichtsmethoden, so raffiniert ausgebaut sie auch immer sein mögen, das klassische Gespräch mit dem Kranken, seiner Lebensgeschichte und seiner Umgebung nicht $\mathrm{zu}$ ersetzen vermögen ${ }^{17}$. Gerade das Verständnis, das Einfühlungsvermögen und die Gesprächsbereitschaft zwischen Arzt und Krankem müssen gelernt werden, und dieses Lernen unterscheidet sich wesentlich vom wissenschaftlichen Studium theoretischer Inhalte ${ }^{8}$. Selbst in den Grundlagefächern bieten sich ethische Fragen an: So soll der Student erkennen, wie weit ein Versuch am Menschen oder am Tier gehen darf, ohne die geltenden ethischen Normen zu verletzen (Immunologie, Mikrobiologie, Pathologie, Pathophysiologie, Pharmakolo- 
gie). Ferner wird der Lernende am Bereich der Prophylaxe (Arbeitsmedizin, Gerichtsmedizin, Sozialmedizin, Versicherungsmedizin) dazu angeleitet, die Freiheit und Ungebundenheit des einzelnen abzuwägen gegenüber den Bedürfnissen und Erfordernissen der Gesellschaft. Als Beispiele bieten sich folgende Themata an: Genußmittel-, Medikamenten- und Drogenmißbrauch, Unfall- und Selbstmordverhütung, Vermeidung von Risikofaktoren zu Krankheit und vorzeitigem Tod.

\section{Praktisches Wahlstudium (in Zürich 5.Studienjahr)}

Während dieser Zeitspanne der Ausbildung steht der Unterassistent besonders nahe am Krankenbett. Er findet Gelegenheit, mitmenschliches Verständnis und Anteilnahme gegenüber dem Leidenden zum Ausdruck zu bringen und sich aus seinem eigenen Egoismus und der Gleichgültigkeit zu lösen ${ }^{8}$. Nicht selten wird er zum eigentlichen Vertrauten des Patienten, der ihm unter Umständen mehr preisgibt als dem Chefarzt oder Assistenten, weil er mehr Zeit für ihn aufbringt und ihm weniger autoritär gegenüber tritt. Gleichzeitig bietet dieses Jahr Gelegenheit, sich im Team eines Spitales einzugliedern und mitmenschliche Kontakte mit den Vorgesetzten, den Kollegen, dem Pflegepersonal und den technischen Spezialisten zu pflegen.

Zusammenfassend sieht Bernheim ${ }^{4}$ die Vermittlung ärztlicher Ethik während der Ausbildung in folgenden drei Stufen: Während der theoretischen Basisausbildung soll Ethik durch einen Lehrer angeboten werden, der gleichzeitig einige rechtliche Grundkenntnisse besitzt und somit neben ethischen auch rechtliche Fragestellungen zu diskutieren vermag. Im Rahmen der verschiedenen Kliniken soll sie durch den entsprechenden Chefarzt vermittelt werden und auf den einzelnen demonstrierten Fall ausgerichtet sein. Schließlich wäre die Ethik im Rahmen des Unterrichts in kleinen Gruppen durch die Lehrbeauftragten oder Assistenten am Bette des einzelnen Kranken darzubieten. Bei allen diesen Gelegenheiten zeigt sich eine enge Verquickung zwischen ethischen Fragen und Rechtsproblemen, weshalb es notwendig und günstig ist, wenn der Student gleichzeitig die wichtigsten rechtlichen Grundlagen seines Handelns kennenlernt. Frühzeitig muß er ferner darauf hingewiesen werden, daß er sich in den entscheidenden Augenblicken seiner Tätigkeit in einer nicht vertretbaren Einsamkeit befindet, in der er, gestützt auf sein fachliches Können, allein auf sein Gewissen gestellt ist ${ }^{11}$. 


\section{Die Unterrichtsmodelle}

Aus der Erkenntnis der nahen Bezüge ethischer und rechtlicher Probleme hat die deutsche Spezialistenordnung die Vermittlung ethisch/rechtlicher Grundlagen dem Gerichtsmediziner überbunden. So lautet der entsprechende Passus: «der Gerichtsmediziner hat sich über Kenntnisse in der Beurteilung der Rechtsstellung des Arztes und der rechtlichen Folgen ärztlichen Handelns auszuweisen». Dementsprechend sollte die gerichtlichmedizinische Vorlesung auch die Behandlung ethischer Fragestellungen beinhalten. An mehreren Medizinschulen existieren schon eigentliche Lehrgänge für medizinische Ethik ${ }^{2,19}$. An der medizinischen Fakultät der Universität Zürich wird seit zehn Jahren eine gemeinsame Vorlesung des Gerichtsmediziners und des Medizinhistorikers zum Thema «Ärztliche Berufs- und Standeskunde» gehalten. Die Gestaltung und Entwicklung dieser Vorlesung, welche vom Studenten im letzten Studienjahr gehört wird, hat gezeigt, wie wertvoll der Beizug des Medizinhistorikers ist. Dieser vermag nämlich sowohl zu rechtlichen als auch zu ethischen Fragestellungen aus der Sicht des Geschichtslehrers, das heißt also des zeitunabhängigen Fachmannes, Stellung zu nehmen. Damit öffnet er dem Studenten den Blick auf die unterschiedlichen Auffassungen zur Ethik und zum Recht bei den verschiedenen Völkern und Kulturen sowie im Ablauf der sich folgenden Zeitepochen. Die Relativität der Gültigkeit einzelner Gesetze und Richtlinien wird damit offenbar. Gleichzeitig soll der Studierende aber auch auf die immer wieder hochgehaltene, humanistisch-christliche Grundhaltung des Arztes im europäischen Kulturkreis hingewiesen werden.

Die beiden Dozenten haben während der bisherigen zehnjährigen Experimentierphase viel gelernt. Vor allem ist es ihnen klar geworden, daß ihre eigenen Kenntnisse als Theoretiker zur Vermittlung einer gültigen und umfassenden praktischen Ethik nicht ausreichen. Deshalb werden neuerdings in zunehmendem Maße entsprechend erfahrene Kollegen verschiedener Fachrichtungen als Gastreferenten beigezogen, wobei nicht so sehr ein Ethiker, Jurist oder Philosoph als vielmehr ein am Krankenbett tätiger, angesehener Arzt gesucht wird. Eine Sonderethik läßt sich für die Ärzteschaft philosophisch ohnehin nicht rechtfertigen. Das ärztliche Ethos ist lediglich als Anwendung allgemeingültiger Verbindlichkeiten der Humanität auf eine besondere Berufssituation zu verstehen ${ }^{8}$. 


\section{Das Zürcher Modell}

Die Vorlesung «Ärztliche Rechts- und Standeskunde» (eine Semesterstunde im letzten Studienjahr) wird derzeit durch folgende Schwerpunkte bestimmt (Detailprogramm siehe Abb. 1):

Abb.1. Zürcher Modell 1982/83

Rechts- und Standeskunde: Vorlesungsprogramm für Medizinstudenten im 6. Studienjahr $(1$ Semester $=16$ Stunden $)$

Praxisbewilligung. Berufsausübung.

Verpflichtung durch Gesetze, Standesordnungen und ethische Richtlinien.

Ärztliche Behandlung und Haftung.

Schweigepflicht mit ihren Ausnahmen.

Zeugnisse und Krankengeschichten.

Forschung am Menschen.

Experimentelle Krebsbehandlung.

Intensivmedizin - Transplantationschirurgie.

Lebenserhaltung von schwerstgeschädigten Neugeborenen.

Begleitung von Schwerkranken.

Sterbehilfe in Praxis und Krankenhaus.

\section{Mündigkeit des Patienten}

Darüber ist in den letzten Jahren viel gesprochen und geschrieben worden. Es sei verwiesen auf die grundlegenden Referate im Rahmen eines Symposiums der Schweizerischen Akademie der medizinischen Wissenschaften vom Sommer 1980 in Basel ${ }^{5}$. Dort diskutierten die Philosophen Jeanne Hersch und Hans Saner, die Theologen Peter Vogelsanger und Albert Ziegler, der Psychologe Charles Durand und der Jurist Hans Schultz mit den Ärzten über die Freiheit, die Würde und die Rechte des Patienten in der heutigen Medizin. Auch Ringeling betont die Personwürde eines jeden Menschen, die nach ihm unbedingt zu gewährleisten ist in der Form der Freiheit, sich selbst zu entfalten, zu verwirklichen und auch zu verändern ${ }^{18}$. Gerade der junge Arzt, beispielsweise als Assistent im Spital, läuft Gefahr, im blinden Vertrauen auf die Fortschritte der Medizin zu wenig auf die Wünsche des Kranken zu achten und dessen Selbstverwirklichung zu behindern. Dies beginnt bereits bei der nur summarisch und unvollständig 
betriebenen Aufklärung, setzt sich fort in der Behandlung ohne oder sogar gegen den Willen des Patienten und endet in der unerwünschten, allenfalls zerstörerischen Veränderung der Persönlichkeit des Kranken im körperlichen, psychischen oder seelischen Bereich. Die Öffentlichkeit ist gegen derartige Verstöße hellhörig geworden. So haben sich Vereinigungen zur Wahrnehmung und zum Schutze der Rechte des Kranken gebildet ${ }^{24}$, und der Student muß wissen, daß überdies in mehreren Kantonen eigentliche Rechtsvorschriften betreffend Umfang und Grenzen der Behandlung eines Patienten im Spital bestehen ${ }^{25}$. Automatisch führen solche Fragestellungen über zu Diskussionen der ärztlichen Haftung, worauf unter Berücksichtigung der neueren Entwicklung der Rechtspraxis eingetreten wird.

\section{Therapeutischer Versuch}

Die Forschung am Menschen ist ganz ähnlich wie die Durchführung von Tierversuchen in den letzten Jahren zunehmend unter Verruf geraten. Dennoch stellt sie einen wesentlichen Bestandteil für die Qualität und den Fortschritt der medizinischen Wissenschaften dar. Damit sie unter ethischen und rechtlichen Gesichtspunkten ohne Kritik durchgeführt werden kann, bedarf sie strikter Richtlinien. Diese haben sich an der Würde des Kranken bzw. des gesunden Forschungsobjektes zu orientieren. Darauf ist bereits in den Schriften des Hippokrates hingewiesen worden ${ }^{10}$. Auch der heutige Medizinstudent muß die Grundlagen dieser Richtlinien kennen, wie sie beispielsweise von der Schweizerischen Akademie der medizinischen Wissenschaften festgelegt wurden ${ }^{14}$. Die medizinische Fakultät der Universität Genf hat sich ferner bemüht, in einem gesamtschweizerischen Kolloquium Einzelprobleme medizinischer Forschung aufzugreifen und zu diskutieren ${ }^{23}$. Der Widerhall vor allem der französischsprechenden Fakultäten war erfreulich. Hingegen stehen die deutschschweizerischen Fakultäten derartigen Aussprachen immer noch zurückhaltend gegenüber. Zu wenig orientierte Wissenschaftler laufen aber Gefahr, bei ihren Forschungen in Zukunft sogar des Rechtsmißbrauches beklagt zu werden, wenn sie sich nicht durch ein ethisches Komitee beraten und kontrollieren lassen ${ }^{12}$.

\section{Arzt-Patienten-Beziehung und öffentliche Interessen}

Dieses Spannungsfeld wirkt sich mit zunehmender Sozialisierung unserer staatlichen Einrichtungen gerade auch auf dem Gebiet der ärztlichen 
Tätigkeit aus. Die einzelnen Standesordnungen befassen sich damit eingehend. Als Beispiel sei die Kostenexplosion genannt, die im Bereich der Spitalmedizin stärker als beim praktizierenden Arzt in Erscheinung tritt. Darauf wird in der Zürcher Vorlesung eingetreten, z. B. bei der Besprechung der Möglichkeiten und Grenzen der Intensivmedizin oder von besonders eingreifenden und kostspieligen ärztlichen Behandlungen (Beispiele: Anwendung von Strahlen oder Zellteilungshemmern bei Krebskranken, Großoperationen in Grenzfällen, Transplantationen, Anschluß an künstlicher Niere, Beatmung am Respirator, Wiederbelebung des Herzens). Die Schweigepflicht, die Meldebefugnisse und die Meldepflichten werden ferner ausführlich diskutiert und gezeigt, daß dabei der Staat in das persönliche Verhältnis zwischen Arzt und Patient eintritt. Aus dem Ich-Du-Bezug wird ein Ich-Es-Bezug ${ }^{1}$. Der Dialog und das enge Vertrauensverhältnis zum Kranken wird aufgebrochen, die Zwischenmenschlichkeit zugunsten eines Gemeinschaftsinteresses beeinträchtigt. Den rechten Weg aus dieser Situation zu finden ist schwierig. Der Arzt soll einerseits nicht als Vertreter des Staates im reinen öffentlichen Interesse handeln; er darf anderseits aber auch nicht bei der Formulierung des Gemeinwohles vollständig beiseite stehen.

\section{Beginn und Ende des Lebens}

Breiten Raum nehmen heute die Diskussionen ein, welche im Zusammenhang mit Genmanipulationen ${ }^{15}$, mit der Beförderung oder der Verhinderung der Nachkommenschaft sowie mit der Pflege und Behandlung mißgebildeter Neugeborener stehen. Am Ende des Lebens stellen sich die Probleme der Sterbehilfe ${ }^{3.16}$. Wir haben es uns zur Pflicht gemacht, diesen Fragestellungen breiten Raum zu widmen und sowohl die Sterbehilfe im Spital als auch in der Praxis erschöpfend zu behandeln. Die Studenten zeigen sich dabei aktiv und äußern sehr unterschiedliche, teilweise kontroverse Auffassungen. Selbst die Richtlinien der Schweizerischen Akademie ${ }^{14}$, so allgemein und klug sie auch gehalten sind, stoßen auf Kritik. Dies ist erwünscht, denn ein wesentlicher Zweck der Vorlesung besteht darin, den Studierenden die Problematik jeder reglementierten und damit starren ethischen Stellungnahme aufzuzeigen. Die verschiedenen möglichen Facetten einer ärztlichen Einstellung sollen ihnen vor Augen geführt werden, damit sie ihre Eigenverantwortlichkeit im konkreten Einzelfall erkennen lernen. 


\section{Zusammenfassung}

Im Medizinstudium muß eine solide naturwissenschaftliche Basis aufgebaut und die Krankheit mit ihren verschiedenen Erscheinungsbildern sowohl systematisch theoretisch als auch im Einzelfall praktisch am Krankenbett vorgezeigt werden. Die Ausbildung soll sich aber auch bemühen, neben dem kundigen Mediziner einen Arzt heranzubilden. Dies bedeutet, daß schon im Studium ethischen Problemen genügend Zeit zu widmen ist. Weit mehr als sonst in den sozialen Beziehungen des Menschen fließen im ärztlichen Berufsbereich ethische mit rechtlichen Problemen zusammen. Deshalb wird in der medizinischen Fakultät der Universität Zürich seit zehn Jahren in einer Semesterstunde des letzten Studienjahrs im Rahmen des gerichtsmedizinischen Unterrichts eine V.orlesung über «Ärztliche Rechts- und Standeskunde» gehalten, welche ein Schwergewicht auf ethische Fragestellungen legt (Abb. 1). Die teilnehmende aktive Reaktion der Studenten ist erfreulich.

\section{Literatur}

${ }^{1}$ Abbt I.: Zwischenmenschlichkeit als Beziehungsvorgang. Schweiz. Ärzte Z. 63 (1982) 2329-2330.

${ }^{2}$ André A.: Notions de déontologie médicale. Fac. méd. Univ., Liège 1982.

${ }^{3}$ Auer A., Menzel H. und Eser A.: Zwischen Heilauftrag und Sterbehilfe. Carl Heymanns Verlag, Köln/Berlin/München 1976.

${ }^{4}$ Bernheim J.: L’enseignement de la déontologie. Fac. méd. Univ., Genève 1980.

${ }^{5}$ Bull. Schweiz. Akad. med. Wiss. 36 (1980) 227-427.

${ }^{6}$ Burns Ch.: Legacies in Ethics and Medicine. Science History Publications, New York 1977.

${ }^{7}$ Gonin D. und Daligand L.: Le médecin, l'éthique et la loi. Méd. et Hyg. 39 (1981) 2621-2624.

${ }^{8}$ Höffe O.: Philosophische Fragmente eines ärztlichen Ethos. Schweiz. Rundschau Med. (Praxis) 70 (1981) 1787-1794.

9 Jaspers K.: Die Idee des Arztes. Schweiz. Ärzte Z. 34 (1953) 253-257.

${ }^{10}$ Koelbing H.: Ist die Menschlichkeit der Medizin selbstverständlich? Schweiz. Ärzte Z. 60 (1979) 1297-1299.

${ }^{11}$ Laufs A.: Recht und Gewissen des Arztes. Heidelberger Jahrb. 24 (1980) 1-15.

${ }^{12}$ Lehmann S.: Zur Frage der rechtlichen Beurteilung von Doppelblindversuchen an Patienten, einschließlich Doppelblindversuche mit Placebo. Schweiz. Ärzte Z. 63 (1982) $2249-2255$.

${ }^{13}$ Lukowsky A.: Zitiert bei Laufs A.

${ }^{14}$ Medizinisch-ethische Richtlinien der Schweiz. Akad. med. Wiss. Schweiz. Ärzte Z. 63 (1982) 615-625.

${ }^{15}$ Milunsky A. und Annas G. J.: Genetics and the law. Plenum Press, New York/London 1975. 
${ }^{16}$ Ostheimer N.C. und J.M.: Life or Death-Who Controls? Springer Publishing Company, New York 1976.

17 Prader A.: «Vorteile unübersehbar.» Uni Zürich 13 (1982) 7, 10-11.

${ }^{18}$ Ringeling H.: Ganzheit und Gesundheit: Biblisches Menschenbild und medizinische Ethik. Schweiz. Ärzte Z. 63 (1982) 2321-2328.

${ }^{19}$ Roy D. J.: L'enseignement de l'éthique médicale. J. méd. lég. droit méd. 25 (1982) 723-732.

${ }^{20}$ Scorer G. und Wing A.: Decision Making in Medecin: The practice of its ethics. Eduard Arnold, Publisher, Bristol 1979.

${ }^{21}$ Stauffenegger M.: Gedanken beim Rücktritt. Schweiz. Ärzte Z. 55 (1974) 77-79.

${ }^{22}$ Streit R.: Gegenwartsprobleme des Ärztestandes. Schweiz. Ärzte Z. 64 (1983) 119-122.

${ }^{23}$ Symposium d'éthique médicale, Fac. méd. Univ. Genève (28.11.1981).

${ }^{24}$ Ulrich W.: Patient, Dein Recht. Mosaik Verlag, Gütersloh 1977.

${ }^{25}$ Verordnung über die zürcherischen kantonalen Krankenhäuser vom 28. Januar 1981.

${ }^{26}$ Wunderli J. und Weisshaupt K.: Medizin im Widerspruch. Walter-Verlag, Olten/Freiburg im Breisgau 1977.

\section{Summary}

Ethics in medical study

During the study a medical student acquires knowledge in natural and medical sciences. $\mathrm{He}$ will be trained in clinical know-how. To become a good doctor he also has to be confronted constantly with medical ethics. This article describes the ethical program of the medical school, University of Zurich, Switzerland (see table 1).

Prof. Dr. med. H.P. Hartmann

Gerichtlich-Medizinisches Institut

Postfach

CH-8028 Zürich 